\title{
PREVALENCE AND FACTORS ASSOCIATED WITH HYPERTENSION AMONG HEALTH WORKERS OF CENTRAL HOSPITALS IN NEPAL
}

\author{
Prakash Ghimire $^{1,2 *}$, Alisha Khadka ${ }^{3}$, Amornrat Anuwatnonthakate ${ }^{1}$, Supaporn Trongsakul ${ }^{1}$ \\ ${ }^{1}$ Department of Public Health, School of Health Science, Mae Fah Luang University, Chiang Rai, Thailand \\ ${ }^{2}$ Department of Health Services, Ministry of Health and Population, Nepal \\ ${ }^{3}$ College of Nursing, Nepalese Army Institute of Health Sciences, Kathmandu, Nepal \\ *Corresponding Author: Prakash Ghimire \\ Email: prakash.ghimire707@gmail.com
}

\begin{abstract}
Hypertension is a significant public health issue considered as the world's biggest killer among the working-age group. Health workers are one of the working groups who are facing several factors in their lives that could induce hypertension among them. Nevertheless, there is a lack of information about hypertension among Nepalese health workers. Therefore, this cross-sectional study aimed to determine the prevalence and factors associated with hypertension among health workers of central hospitals in Nepal. A total of 422 health workers aged $\geq 30$ years from seven central hospitals of Nepal were selected using a proportionate stratified systematic random sampling method. Information regarding characteristics (socio-demographic, clinical, behavioral, occupational, and perceived stress) of participants were obtained by face to face interviews using a questionnaire after getting informed written consent. Participant's blood pressure and body mass index were measured based on the World Health Organization STEP Surveillance Manual. Binary logistic regression model was used to identify the associated factors of hypertension. The prevalence of hypertension was 35.31\% (95\% CI: 30.70\%-40.10\%). After controlling potential confounding factors through multivariate analysis, seven factors (increasing age, low educational level, being married, low fruit \& vegetable consumption, low physical activity, alcohol use, and increased perceived stress) were found as significant independent predictors of hypertension. In conclusion, hypertension is highly prevalent among Nepalese health workers. Effective behavioral change interventions and routine health screening programs focusing on hypertension should be implemented to control hypertension among this neglected group, Nepalese health workers.
\end{abstract}

Keywords: Health Workers, Hypertension, Perceived stress, Physical activity, Prevalence, Nepal

\section{INTRODUCTION}

Hypertension is a growing global public health concern responsible for substantial morbidity and mortality, primarily affecting the working-age population of low and middle-income countries (World Health Organization, 2018). Hypertension is the topmost preventable non-communicable disease (NCD), as well as a risk factor of NCDs that embraced 9.4 million deaths, more than the deaths from infectious illnesses combined (International Federation of Pharmaceutical Manufacturers and Associations, 2016; World Health Organization, 2018).The worldwide prevalence of hypertension among adult people aged $\geq 18$ years was around 22\% in 2015 (World Health Organization, 2018). In the South-East
Asian Region (SEAR), hypertension accounted for 1.5 million deaths each year and found that one in three adults has high blood pressure (World Health Organization, 2013). A meta-analysis revealed the high prevalence of hypertension $(33.80 \%)$ in Nepal as compared to other SAARC (South Asian Association for Regional Cooperation) countries (Neupane et al., 2014). Nepal is a landlocked, least developed country situated in the SEAR currently facing a triple burden of disease, including overwhelming consequences of $\mathrm{NCD}$, significantly hypertension.

Based on the Nepal Step Survey 2013, the hypertension rate in the Nepalese adult people was $25.70 \%$ (Aryal et al., 2014). Furthermore, the pooled analysis of hypertension prevalence rate among the adult population of diverse geographical 
locations in Nepal was ranged from $15.1 \%$ to $38.9 \%$ (Dhungana et al., 2016; Khanal et al., 2017; Neupane et al., 2017). The working-age adult population was the victim of 12.2 million premature deaths from NCD, mainly hypertension worldwide (World Health Organization, 2017). Health workers are an essential cohort of the nation that belongs to the working-age group. As health guardians, health workers are health role models for their patients, families, and communities.

The health of health workers is of great significance because they must be healthy for their better job performance. Unfortunately, the working conditions of health workers expose them to long working duration, shift duties, unhealthy dietary habits, sedentary lifestyle, and stress, which are the significant inducers of hypertension (Taylor and Bithoney, 2012; Manyisa and Aswegen, 2017; World Health Organization, 2017; Muzzi, Pawlina and Schnorr, 2018). Hypertension is not only responsible for deaths among health workers but also accountable for their disability, poor mental health and quality of life, an increased burden in health expenditure of the nation, altered health system and its effects on patients, low workplace productivity from their absence (Taylor and Bithoney, 2012; World Health Organization, 2013; Kretchy, Owusu-daaku and Danquah, 2014; Asay et al., 2016; International Federation of Pharmaceutical Manufacturers and Associations, 2016). Several studies evident the momentous upsurge of hypertension and further cardiovascular disease risk factors in health workers in comparison to other working groups (Sumaila et al., 2016). Few studies show the prevalence of hypertension among health workers at $9.20 \%$ in India, $8.60 \%$ in Iran, and $35.27 \%$ in Thailand, respectively (Sahebi, Vahidi and Mousavi, 2010; Ahmed, Jadhav and Sobagaiah, 2018; Sirinara, Hanprathet and Jiamjarasrangsi, 2019). Nonetheless, until now, no studies have been conducted, which shows the direct figure of hypertension among health workers in Nepal. Therefore, a study is necessary to uncover the concealed situation of hypertension among Nepalese health workers.

Health workers are the pillar of the health system in Nepal. The majority of the Nepalese health workers $(23.00 \%)$ with diverse job cadre are working in the central level hospitals (Ministry of Health and Population, 2013). These hospitals have a high burden of workload, sedentary work style, deprived sleep, and shift work as compared to other levels of health organizations in Nepal (Thapa, Malla and $\mathrm{KC}, 2018$ ). To date, there is no provision of health screening programs focusing on hypertension for Nepalese health workers, due to which the Nepalese government is unaware of their health situation and thought that their health is not of priority concern.

Thus, this study aimed to estimate the prevalence and determine the factors associated with hypertension among health workers of central hospitals in Nepal, which could facilitate the formulation of effective health intervention to control the foreseeable burden of hypertension in a neglected group, Nepalese health workers.

\section{METHODS}

An analytical cross-sectional study was conducted among health workers in seven central hospitals located in Lalitpur and Kathmandu districts of Bagmati Province, Nepal, from September to November 2019. Central hospitals are the tertiary level health organization under the health system of Nepal. In 2019, there were eleven central hospitals, according to the ministry of health and population, Nepal. Out of them, seven central hospitals were included as the study settings in this study as they consist of a large number of varieties of health workers working and dealing with the high flow of patients and workload (Ministry of Health and Population, 2013).

Based on the reports from 
respective central hospitals, there was a 1,942 study population working in the study settings on the date of study in 2019 . All the health workers aged $\geq 30$ years working in central hospitals for at least one year were inclusion criteria of this study. Whereas, pregnant, who were unable to respond, and on leave or training were the exclusion criteria.

$\begin{array}{cc}\text { After using Cochran formula }(\mathrm{n}= \\ \left.\mathrm{Z}^{2} \mathrm{pq} / \mathrm{e}^{2}\right) ; \quad \mathrm{Z}(\text { desired } & \text { confidence }\end{array}$ interval $)=1.96, \mathrm{p}($ assumed prevalence $)=0.5$ (Naing, Winn and Rusli, 2006), $\mathrm{q}=0.5$, $\mathrm{e}$ (allowable error) $=0.05$ and adding $10 \%$ to account any errors, the total required sample size was estimated at 422. The appropriate sample was chosen using a proportionate stratified systematic random sampling method where strata refer to the types of health workers (doctors, nursing staff, paramedic staff, and other health staff). Firstly, the proportion of the required sample size $(21.73 \%)$ inside the stratum of each central hospital was determined. Then, inside each stratum, the first sample was taken randomly, followed by the consecutive sample at each $5^{\text {th }}$ interval until getting the required number of samples from respective hospitals (Figure 1).

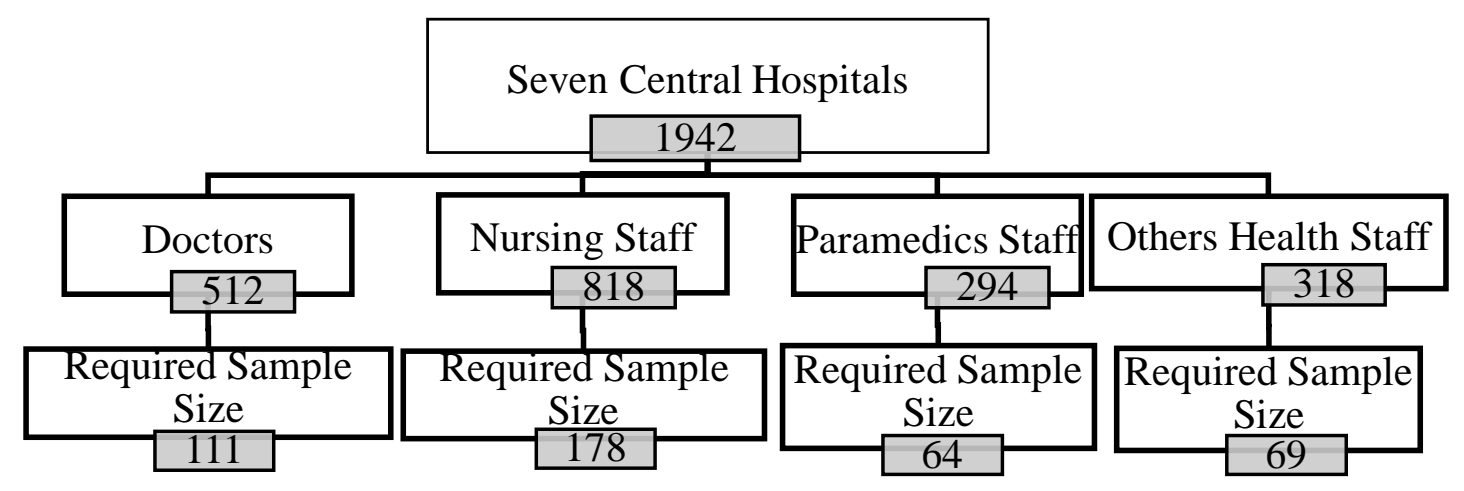

Figure 1. Flowchart of sample selection

A questionnaire was developed based on the WHO STEP Survey Questionnaire, Cohen's Perceived Stress Scale-10, and literature review. Questionnaire's internal validity was evaluated by three Nepalese experts based on IOC method and found each item of questionnaire as congruent (IOC score $>0.5)$. A translated Nepali version questionnaire was piloted in 30 randomly selected health workers of tertiary hospital; other than study settings, to check its face validity and reliability. The internal consistency of the perceived stress-related questions was tested and found Cronbach's alpha value at 0.746 . Then, the information about socio-demographic characteristics (age, sex, educational level, marital status, monthly family income, type of health workers, employment status), clinical characteristics (family history of hypertension, history of known NCDs) behavioral characteristics (amount of salt intake, added salt intake, fruits \& vegetable consumption, physical activity level, alcohol use, tobacco use), occupational characteristics (work duration, shift work, sleep duration), and perceived stress of the participants were obtained by face-faceinterviews using a questionnaire. The physical measurement tools (digital BP machine, digital weighing machine, and portable standard stature) were identified, calibrated, and used to measure blood pressure, height, and weight of the participants based on the WHO STEP Surveillance Manual (Aryal et al., 2014).

Three readings of blood pressure were taken from participants and categorized as hypertensive if the mean of last two readings has $\geq 140 \mathrm{mmHg}$ systolic blood pressure or/and $\geq 90 \mathrm{mmHg}$ diastolic 
blood pressure or had a self-reported history of hypertension (Chobanian et al., 2003; Aryal et al., 2014).

Body mass index was calculated using the formula, $\mathrm{BMI}=$ weight $(\mathrm{kg}) /$ height $\left(\mathrm{m}^{2}\right)$ and classified as underweight $(<18.50)$, normal (18.50-24.99), and Obese ( $\geq 30)$. Physical activity level was assessed by selfreported duration and intensity of physical activity per week, later converted into Metabolic Equivalent Task (MET)minute/week. METs-value for moderateintensity activity and vigorous-intensity activity was 4 MET/minute and 8 $\mathrm{MET} / \mathrm{minute}$. The normal physical activity level was adopted as $\geq 600$ METminute/week. The consumption of fruits and vegetables less than five servings per day termed as Low consumption. Individuals who used alcohol products (at least 50 grams) and tobacco products in their lifetime were referred as alcohol users and tobacco users, respectively. Regarding perceived stress, participants were asked ten questions (four positive and six negative) and labelled the score (0-4) of responses to each question. Then, the total score was categorized as $(0-13)=$ low perceived stress, $(14-26)=$ moderate perceived stress, and $(27-40)=$ high perceived stress (Cohen and Williamson, 1988; NH Department of Administrative Services, 2014).

Data were compiled, coded, entered, cleaned, and analyzed in SPSS V.20.0. Descriptive statistics were used to describe the characteristics of the participants. All the categorical data were presented as frequency and percentage, while continuous data as mean with standard deviation. Hypertension prevalence was illustrated in percentage with 95\% Confidence Interval (CI). The associated factors from univariate analysis at $p$-value 0.2 were entered into the multiple logistic regression model to detect the significant independent factors of hypertension with Adjusted Odds Ratio (AOR) at 95\% CI and p-value $<0.05$.

The ethical review board of Mae Fah Luang University (REH-62164) and
Nepal Health Research Council (Reg. no. 696/2019) approved this study based on the Helsinki Declaration 2002. The written permission for data collection was obtained from the study settings. The informed written consent was taken from the participants prior to the data collection and confidentially was maintained thoroughly.

\section{RESULTS}

A total of 422 health workers were enrolled with a $100 \%$ response rate in the study. The mean ( \pm standard deviation) age of the participants was $36.50( \pm 5.77)$ years old. Majority of the participants were aged $30-39$ years $(59.70 \%)$, female $(58.80 \%)$, graduate $(36.00 \%)$, married $(82.50 \%)$, and nursing staff (42.20\%). 16.40\% were other health staff (pharmacy staff, laboratory staff, radiological staff, anesthesia staff, dietetics staff, ophthalmic staff, and dental staff). Similarly, $38.60 \%$ of participants had a monthly family income of 51000-80000 NRs, and $75.80 \%$ had permanent employment.

Half of the participants $(50.90 \%)$ had a family history of hypertension, whereas only $13.27 \%$ of participants found to have a history of diabetes. $11.37 \%$ of the participants had history of other NCDs (kidney disease, hypothyroidism, hyperthyroidism, arthritis, asthma, gout, hemorrhoids, hyperlipidemia, and migraine). More than half of the participants were overweight $(52.80 \%)$. $66.80 \%$ of participants had a habit of low fruits and vegetable consumption $(<5$ servings/day). The minority of the participants $(31.80 \%)$

consumed the recommended amount of salt ( $<5$ grams/day). Furthermore, only $37.70 \%$ of participants used added salt before and during eating food. $62.80 \%$ of participants found to be less physically active $(<600$ MET-minute/week). Majority of the participants $(59.20 \%)$ consumed at least one standard drink of alcohol in their lifetime, while only $27.50 \%$ of participants found to use tobacco products. Most of the 
participants were working on a rotating shift basis $(53.60 \%)$ and had a work duration of $1-9$ years $(55.70 \%) .85 .10 \%$ of participants had normal sleep duration $(\geq 6$ hours/day). Regarding the perceived stress, more than $3 / 5^{\text {th }}$ of the participants $(62.60 \%)$ perceived a moderate level of stress (Table 1). The proportion of hypertension was higher among participants with age 40-49 years, female, post-graduate or higher education, married, nursing staff, family history of hypertension, overweight, low fruits \& vegetable consumption, high salt intake (5-10 grams/day), low physical activity, alcohol use and a moderate level of perceived stress (Table 1).

Table 1. Characteristics of hypertensive and non-hypertensive participants

\begin{tabular}{|c|c|c|c|}
\hline \multirow{3}{*}{ Characteristics } & \multirow{3}{*}{ Total n (\%) } & \multicolumn{2}{|c|}{ Hypertension } \\
\hline & & Yes & No \\
\hline & & n (\%) & n (\%) \\
\hline Total & $422(100.00)$ & $149(35.31)$ & $273(64.69)$ \\
\hline \multicolumn{4}{|c|}{ Socio-demographic characteristics } \\
\hline \multicolumn{4}{|l|}{ Age (years) } \\
\hline $30-39$ & $252(59.70)$ & $44(29.53)$ & $208(76.19)$ \\
\hline $40-49$ & $137(32.50)$ & $81(54.36)$ & $56(20.51)$ \\
\hline $50-59$ & $33(7.80)$ & $24(16.11)$ & $9(3.30)$ \\
\hline \multicolumn{4}{|c|}{ mean $\pm \mathrm{SD}=36.50 \pm 5.77$} \\
\hline \multicolumn{4}{|l|}{ Sex } \\
\hline Male & $174(41.20)$ & $74(49.66)$ & $100(36.63)$ \\
\hline Female & $248(58.80)$ & $75(50.34)$ & $173(63.37)$ \\
\hline \multicolumn{4}{|l|}{ Level of education } \\
\hline Technical School & $41(9.70)$ & $14(9.39)$ & $27(9.89)$ \\
\hline \multicolumn{4}{|c|}{$\begin{array}{l}\text { Leaving Certificate } \\
\text { (TSLC) }\end{array}$} \\
\hline Proficiency & $108(25.60)$ & $36(24.16)$ & $73(26.74)$ \\
\hline \multicolumn{4}{|l|}{$\begin{array}{l}\text { Certificate Level } \\
\text { (PCL) }\end{array}$} \\
\hline Graduate & $152(36.00)$ & $49(32.88)$ & $102(37.36)$ \\
\hline $\begin{array}{l}\text { Post-graduate or } \\
\text { higher }\end{array}$ & $121(28.70)$ & $50(33.57)$ & $71(26.01)$ \\
\hline \multicolumn{4}{|l|}{ Marital status } \\
\hline Unmarried & $52(12.30)$ & $4(2.68)$ & $48(17.58)$ \\
\hline Married & $348(82.50)$ & $134(89.93)$ & $214(78.39)$ \\
\hline Separated/ & $12(5.20)$ & $11(7.39)$ & $9(4.03)$ \\
\hline \multicolumn{4}{|l|}{ Divorced and } \\
\hline \multicolumn{4}{|l|}{ Widowed } \\
\hline \multicolumn{4}{|c|}{ Monthly family income (NRs) } \\
\hline $20000-50000$ & $103(24.40)$ & $29(19.46)$ & $74(27.11)$ \\
\hline $51000-80000$ & $163(38.60)$ & $50(33.56)$ & $113(41.39)$ \\
\hline $81000-110000$ & $81(19.20)$ & $30(20.13)$ & $51(18.68)$ \\
\hline$>110000$ & $75(17.80)$ & $40(26.85)$ & $35(12.82)$ \\
\hline \multicolumn{4}{|l|}{ Type of health worker } \\
\hline Doctors & $112(26.50)$ & $41(27.52)$ & $71(26.01)$ \\
\hline Nursing staff & $178(42.20)$ & $59(39.60)$ & $119(43.59)$ \\
\hline Paramedic staff & $63(14.90)$ & $22(14.76)$ & $41(15.02)$ \\
\hline Other health staff & $69(16.40)$ & $27(18.12)$ & $42(15.38)$ \\
\hline
\end{tabular}




\begin{tabular}{|c|c|c|c|}
\hline \multirow{3}{*}{ Characteristics } & \multirow{3}{*}{ Total n (\%) } & \multicolumn{2}{|c|}{ Hypertension } \\
\hline & & Yes & No \\
\hline & & n (\%) & n (\%) \\
\hline \multicolumn{4}{|l|}{ Employment status } \\
\hline Permanent & $320(75.80)$ & $128(85.91)$ & $192(70.33)$ \\
\hline Contract & $70(16.60)$ & $14(9.39)$ & $56(20.51)$ \\
\hline Temporary & $32(7.60)$ & $7(4.70)$ & $25(9.16)$ \\
\hline \multicolumn{4}{|c|}{ Clinical characteristics } \\
\hline \multicolumn{4}{|c|}{ Family history of hypertension } \\
\hline Yes & $215(50.90)$ & $98(65.77)$ & $117(42.86)$ \\
\hline No & $186(44.10)$ & $43(28.86)$ & $143(52.38)$ \\
\hline Unknown & $21(5.00)$ & $8(5.37)$ & $13(4.76)$ \\
\hline \multicolumn{4}{|l|}{ History of diabetes } \\
\hline Yes & $56(13.27)$ & $36(24.16)$ & $20(7.33)$ \\
\hline No & $366(86.73)$ & $113(75.84)$ & $253(92.67)$ \\
\hline \multicolumn{4}{|c|}{ History of other NCD } \\
\hline Yes & $48(11.37)$ & $18(12.08)$ & $30(10.99)$ \\
\hline No & $374(88.63)$ & $131(87.92)$ & $248(89.01)$ \\
\hline \multicolumn{4}{|l|}{ Body Mass Index } \\
\hline Normal & $184(43.60)$ & $30(20.13)$ & $154(56.41)$ \\
\hline Overweight & $223(52.80)$ & $109(73.15)$ & $114(41.76)$ \\
\hline Obesity & $15(3.60)$ & $10(6.72)$ & $5(1.83)$ \\
\hline \multicolumn{4}{|c|}{ Behavioral characteristics } \\
\hline \multicolumn{4}{|c|}{ Fruit and vegetable consumption (servings/day) } \\
\hline$<5$ & $282(66.80)$ & $129(86.58)$ & $153(56.04)$ \\
\hline$\geq 5$ & $140(33.20)$ & $20(13.42)$ & $120(43.96)$ \\
\hline \multicolumn{4}{|c|}{ Amount of salt intake (grams/day) } \\
\hline$<5$ & $134(31.80)$ & $26(17.45)$ & $108(39.56)$ \\
\hline $5-10$ & $228(54.00)$ & $88(59.06)$ & $140(51.28)$ \\
\hline$>10$ & $60(14.20)$ & $35(23.49)$ & $25(9.16)$ \\
\hline \multicolumn{4}{|l|}{ Added salt intake } \\
\hline Yes & $159(37.70)$ & $70(46.98)$ & $89(32.60)$ \\
\hline No & $263(62.30)$ & $79(53.02)$ & $184(67.40)$ \\
\hline \multicolumn{4}{|c|}{ Level of physical activity (MET-minutes/week) } \\
\hline$<600$ & $265(62.80)$ & $123(82.55)$ & $142(52.01)$ \\
\hline$\geq 600$ & $157(37.20)$ & $26(17.45)$ & $131(47.99)$ \\
\hline \multicolumn{4}{|l|}{ Tobacco use } \\
\hline Yes & $116(27.50)$ & $69(46.31)$ & $47(17.22)$ \\
\hline No & $306(72.50)$ & $80(53.69)$ & $226(82.78)$ \\
\hline \multicolumn{4}{|l|}{ Alcohol use } \\
\hline Yes & $250(59.20)$ & $132(88.59)$ & $118(43.22)$ \\
\hline No & $172(40.80)$ & $17(11.41)$ & $155(56.78)$ \\
\hline \multicolumn{4}{|c|}{ Occupational characteristics } \\
\hline \multicolumn{4}{|l|}{ Shift work } \\
\hline Day (non-shift) & $155(36.70)$ & $65(43.62)$ & $90(32.96)$ \\
\hline Morning shift & $41(9.70)$ & $19(12.76)$ & $22(8.06)$ \\
\hline Rotating shift & $226(53.60)$ & $65(43.62)$ & $161(58.98)$ \\
\hline \multicolumn{4}{|l|}{ Work duration (years) } \\
\hline $1-9$ & $235(55.70)$ & $47(31.54)$ & $188(68.87)$ \\
\hline $10-19$ & $123(29.10)$ & $62(41.61)$ & $61(22.34)$ \\
\hline
\end{tabular}




\begin{tabular}{|c|c|c|c|}
\hline \multirow{3}{*}{ Characteristics } & \multirow{3}{*}{ Total n (\%) } & \multicolumn{2}{|c|}{ Hypertension } \\
\hline & & Yes & No \\
\hline & & n (\%) & n (\%) \\
\hline$\geq 20$ & $64(15.20)$ & $40(26.85)$ & $24(8.79)$ \\
\hline \multicolumn{4}{|c|}{ Sleep duration (hours/day) } \\
\hline$<6$ & $63(14.90)$ & $49(32.89)$ & $14(5.13)$ \\
\hline$\geq 6$ & $359(85.10)$ & $100(67.11)$ & $259(94.87)$ \\
\hline \multicolumn{4}{|c|}{ Level of perceived stress (score) } \\
\hline Low $(0-13)$ & $144(34.10)$ & $20(13.43)$ & $124(45.42)$ \\
\hline Moderate (14-26) & $264(62.60)$ & $118(79.19)$ & $146(53.48)$ \\
\hline High $(27-40)$ & $14(3.30)$ & $11(7.38)$ & $3(1.10)$ \\
\hline
\end{tabular}

The prevalence of hypertension among health workers was $35.31 \%$ (95\% CI: 30.70-40.10). Out of those with hypertension, $79(53.02 \%)$ participants had diagnosed hypertension, whereas $70(46.98 \%)$ participants had undiagnosed hypertension. Furthermore, almost half of the participants $39 \quad(49.40 \%)$ with diagnosed hypertension, were not able to control their hypertension.

In multivariate analysis, the significantly associated factors of hypertension were age, educational level, marital status, fruits \& vegetable consumption, physical activity, alcohol use, and perceived stress. The results showed that health workers who fell under age group 40-49 years and age group 50-59 years were 3.11 times (AOR:3.11, 95\% CI:1.38-6.97), and 6.18 times (AOR:6.18, 95\% CI:1.51-25.30) more likely to get hypertension respectively as compared to health workers aged 30-39 years. The health workers who had the Technical School Leaving Certificate (TSLC) level of education were 4.58 times (AOR:4.58, 95\% CI:1.44-14.62), more likely to have hypertension as compared to participants who had a post-graduate or higher level of education. The chance of having hypertension in married health workers was 4.34 times (AOR:4.34, 95\% CI:1.85-15.90) as compared to unmarried health workers. Health workers who consumed $<5$ servings of fruits and vegetable per day were 2.27 times (AOR:2.27, 95\% CI:1.06-4.88), more likely to get hypertension than those who consumed $\geq$ five servings of fruits and vegetable per day. Similarly, health workers with low physical activity $(<600$ METminutes/week) were 2.49 times (AOR:2.49, 95\% CI:1.12-5.14), more likely to be hypertensive as compared to those with normal physical activity $(\geq 600$ METminutes/week). Moreover, among alcohol users, the odds of having hypertension had increased by 4.57 times (AOR:4.57, 95\% CI:2.11-9.92) than non-alcohol drinkers at all. Additionally, health workers who had moderate perceived stress and high perceived stress were 2.64 times (AOR:2.64, 95\% CI:1.26-5.54) and 15.09 times (AOR:15.09, 95\% CI:2.27-100.22) more likely to have hypertension respectively than health workers who had low perceived stress (Table 2).

Table 2. Univariate and multivariate analysis of factors associated with hypertension

\begin{tabular}{ccccc}
\hline Factors & $\begin{array}{c}\text { Crude OR } \\
(\mathbf{9 5 \%} \text { CI) }\end{array}$ & p-value & $\begin{array}{c}\text { Adjusted OR } \\
\mathbf{( 9 5 \% C I )}\end{array}$ & p-value \\
\hline Age (in years) & Reference & & Reference & \\
$30-39$ & $6.84(4.27-10.95)$ & $<0.001$ & $3.11(1.38-6.97)$ & 0.006 \\
$40-49$ & $12.61(5.48-28.97)$ & $<0.001$ & $6.18(1.51-25.30)$ & 0.011 \\
$50-59$ & & & & \\
Sex & $1.71(1.14-2.56)$ & 0.010 & $1.05(0.49-2.26)$ & 0.897
\end{tabular}




\begin{tabular}{|c|c|c|c|c|}
\hline Factors & $\begin{array}{c}\text { Crude OR } \\
(95 \% \text { CI })\end{array}$ & p-value & $\begin{array}{c}\text { Adjusted OR } \\
(95 \% \mathrm{CI})\end{array}$ & p-value \\
\hline Female & Reference & & Reference & \\
\hline \multicolumn{5}{|l|}{ Education level } \\
\hline TSLC & $0.74(0.35-1.54)$ & 0.417 & $4.58(1.44-14.62)$ & 0.010 \\
\hline PCL & $0.71(0.41-1.22)$ & 0.213 & $2.37(0.96-5.85)$ & 0.061 \\
\hline Graduate & $0.68(0.41-1.11)$ & 0.122 & $1.87(0.82-4.25)$ & 0.134 \\
\hline $\begin{array}{l}\text { Post-graduate } \\
\text { or higher }\end{array}$ & Reference & & Reference & \\
\hline \multicolumn{5}{|l|}{ Marital status } \\
\hline Unmarried & Reference & & Reference & \\
\hline Married & $7.14(2.65-21.31)$ & $<0.001$ & $4.34(1.85-15.90)$ & 0.027 \\
\hline $\begin{array}{l}\text { Separated/ } \\
\text { divorced and } \\
\text { widowed }\end{array}$ & $12.00(3.21-44.86)$ & $<0.001$ & $2.59(0.44-15.28)$ & 0.293 \\
\hline \multicolumn{5}{|c|}{ Monthly family income (NRs) } \\
\hline $20000-50000$ & Reference & & Reference & \\
\hline $51000-80000$ & $1.13(0.66-1.94)$ & 0.661 & $0.61(0.26-1.41)$ & 0.251 \\
\hline $81000-110000$ & $1.50(0.80-2.80)$ & 0.201 & $0.85(0.31-2.31)$ & 0.757 \\
\hline$>110000$ & $2.92(1.56-5.45)$ & 0.001 & $1.26(0.45-3.67)$ & 0.665 \\
\hline \multicolumn{5}{|c|}{ Type of health worker } \\
\hline Nursing staff & Reference & & & \\
\hline Doctors & $1.16(0.71-1.91)$ & 0.546 & & \\
\hline Paramedic staff & $1.08(0.59-1.98)$ & 0.798 & & \\
\hline $\begin{array}{l}\text { Other health } \\
\text { staff }\end{array}$ & $1.29(0.73-2.30)$ & 0.376 & & \\
\hline \multicolumn{5}{|c|}{ Type of employment } \\
\hline Permanent & Reference & & Reference & \\
\hline Contract & $0.37(0.20-0.70)$ & 0.002 & $0.91(0.35-2.35)$ & 0.840 \\
\hline Temporary & $0.42(0.18-1.00)$ & 0.050 & $3.40(0.99-11.58)$ & 0.050 \\
\hline \multicolumn{5}{|c|}{ Family history of hypertension } \\
\hline Yes & $2.79(1.80-4.30)$ & $<0.001$ & $1.49(0.80-2.79)$ & 0.211 \\
\hline Unknown & $2.05(0.80-5.26)$ & 0.137 & $1.43(0.37-5.57)$ & 0.604 \\
\hline No & Reference & & Reference & \\
\hline \multicolumn{5}{|l|}{ Known diabetes } \\
\hline Yes & $4.03(2.23-7.27)$ & $<0.001$ & $1.26(0.54-2.95)$ & 0.589 \\
\hline No & Reference & & Reference & \\
\hline \multicolumn{5}{|c|}{ Known kidney disease } \\
\hline Yes & $1.10(0.26-4.67)$ & 0.896 & & \\
\hline No & Reference & & & \\
\hline \multicolumn{5}{|l|}{ Other known NCD } \\
\hline Yes & $1.11(0.57-2.18)$ & 0.761 & & \\
\hline No & Reference & & & \\
\hline \multicolumn{5}{|l|}{ Body mass index } \\
\hline Normal & Reference & & Reference & \\
\hline Overweight & $4.91(3.06-7.86)$ & $<0.001$ & $1.58(0.81-3.05)$ & 0.176 \\
\hline Obesity & $10.27(3.27-32.18)$ & $<0.001$ & $4.49(0.99-20.36)$ & 0.051 \\
\hline
\end{tabular}

Fruits and vegetable consumption (servings/day) 


\begin{tabular}{|c|c|c|c|c|}
\hline Factors & $\begin{array}{c}\text { Crude OR } \\
(95 \% \text { CI })\end{array}$ & p-value & $\begin{array}{c}\text { Adjusted OR } \\
(95 \% \mathrm{CI})\end{array}$ & p-value \\
\hline$<5$ & $5.06(2.98-8.58)$ & $<0.001$ & $2.27(1.06-4.88)$ & 0.035 \\
\hline$\geq 5$ & Reference & & Reference & \\
\hline \multicolumn{5}{|c|}{ Amount of salt intake (gram/day) } \\
\hline$<5$ & Reference & & Reference & \\
\hline $5-10$ & $2.61(1.58-4.32)$ & $<0.001$ & $1.40(0.67-2.93)$ & 0.376 \\
\hline$>10$ & $5.81(2.98-11.35)$ & $<0.001$ & $2.02(0.73-5.63)$ & 0.178 \\
\hline \multicolumn{5}{|l|}{ Added salt intake } \\
\hline Yes & $1.82(1.22-2.76)$ & 0.004 & $0.82(0.43-1.57)$ & 0.549 \\
\hline No & Reference & & Reference & \\
\hline \multicolumn{5}{|c|}{ Physical activity (METminutes/week) } \\
\hline$<600$ & $4.36(2.69-7.09)$ & $<0.001$ & $2.49(1.12-5.14)$ & 0.013 \\
\hline$\geq 600$ & Reference & & Reference & \\
\hline \multicolumn{5}{|l|}{ Tobacco use } \\
\hline Yes & $4.15(2.64-6.50)$ & $<0.001$ & $1.23(0.57-2.68)$ & 0.585 \\
\hline No & Reference & & Reference & \\
\hline \multicolumn{5}{|l|}{ Alcohol use } \\
\hline Yes & $10.20(5.83-17.84)$ & $<0.001$ & $4.57(2.11-9.92)$ & $<0.001$ \\
\hline No & Reference & & Reference & \\
\hline \multicolumn{5}{|c|}{ Work duration (years) } \\
\hline $0-9$ & Reference & & Reference & \\
\hline $10-19$ & $4.06(2.52-6.55)$ & $<0.001$ & $1.46(0.66-3.23)$ & 0.346 \\
\hline$\geq 20$ & $6.66(3.66-12.13)$ & $<0.001$ & $1.22(0.40-3.77)$ & 0.726 \\
\hline \multicolumn{5}{|l|}{ Shift work } \\
\hline Day work & Reference & & Reference & \\
\hline Morning shift & $1.19(0.60-2.39)$ & 0.612 & $1.46(0.55-3.88)$ & 0.442 \\
\hline Rotating shift & $0.56(0.36-0.86)$ & 0.008 & $0.66(0.34-1.31)$ & 0.236 \\
\hline \multicolumn{5}{|c|}{ Sleep duration (hours per day) } \\
\hline$<6$ & $9.06(4.79-17.14)$ & $<0.001$ & $1.85(0.77-4.43)$ & 0.168 \\
\hline$\geq 6$ & Reference & & Reference & \\
\hline \multicolumn{5}{|c|}{ Level of perceived stress (score) } \\
\hline Low $(0-13)$ & Reference & & Reference & \\
\hline $\begin{array}{l}\text { Moderate } \\
(14-26)\end{array}$ & $5.01(2.94-8.52)$ & $<0.001$ & $2.64(1.26-5.54)$ & 0.010 \\
\hline High (27-40) & $22.73(5.83-88.68)$ & $<0.001$ & $15.09(2.27-100.22)$ & 0.005 \\
\hline
\end{tabular}

\section{DISCUSSION}

It is the first epidemiological study that demonstrates the prevalence of hypertension with its associated factors among Nepalese health workers. The prevalence of hypertension among health workers in the current study was $35.31 \%$. This prevalence is higher than the previous studies in India (9.20\%), Iran (8.60\%), and Ghana (16.07\%) (Sahebi, Vahidi and
Mousavi, 2010; Ahmed, Jadhav and Sobagaiah, 2018; Osei-yeboah et al., 2018). It could be possible due to that previous studies has included individual aged $<30$ years, excluded the individual with hypertension and diabetes, and different study settings; primary care center of rural areas (Sahebi, Vahidi and Mousavi, 2010; Ahmed, Jadhav and Sobagaiah, 2018; Oseiyeboah et al., 2018). Besides, one study had an annual health screening program for 
health workers, which could promote awareness among health care workers and led to a low prevalence of hypertension when comparing to this study (Osei-yeboah et al., 2018). The study from Thailand demonstrates prevalence $(35.27 \%)$ consistent with the current finding, where the analogous working environment of participants (in tertiary hospitals of urban areas) might be a reason behind it (Sirinara, Hanprathet and Jiamjarasrangsi, 2019). Aryal et al (2014) reported $36.60 \%$ of hypertension prevalence among Nepalese adult population aged $\geq 30$ years, which is slightly higher than the current result. Comparing to the general population, it could be possible that health workers may have higher profound health knowledge, which leads to better health behaviours and disease prevention.

In this study, increasing age was significantly associated with hypertension. A similar result was reported by previous studies among health workers in Nigeria, Thailand, and Ghana (Sumaila et al., 2016; Osei-yeboah et al., 2018; Sirinara, Hanprathet and Jiamjarasrangsi, 2019). Nepal demographic health survey 2016 revealed the same finding that increasing age was significantly associated with hypertension among Nepalese adults (Kibria et al., 2018). It was physiologically evident that older people are inevitable to get hypertension, a result of gradual changes in the cardiovascular system; vascular remodelling, arterial stiffness, and endothelial dysfunction (Alghatrif et al., 2013).

A current study found that health workers having TSLC level of education were at high risk of hypertension than health workers with post-graduate or higher education. Health workers with TSLC education tended to have limited health knowledge as they learned only 18 months of basic health courses (Council For Technical Education and Vocational Training, 2020). Furthermore, analysis of current findings revealed that health workers with TSLC education were less physically active, use alcohol, consume less fruits and vegetables, and had increased perceived stress, i.e., known inducers of hypertension. Similarly, low education was significantly associated with hypertension among Iranian health workers and Nepalese adults (Sahebi, Vahidi and Mousavi, 2010; Chataut, Adhikari and Sinha, 2011). A consistent result was indicated in the past research (Schwenk, 2017).

The current study showed that being married was significantly associated with hypertension among health workers. A similar finding was noted from previous studies in Nepalese adult population and health workers (Sahebi, Vahidi and Mousavi, 2010; Dhungana et al., 2016). Nepalese married people, especially females in urban areas, have busy and complicated life boost them to acquire stressful life, low physical activity, and unhealthy eating behaviours such as junk foods rather than fruits and vegetables (Ban, 2018). A research evidence back-up the current result by describing that the transition from single to married life possesses lifestyle modification such as sedentary lifestyle, which adversely impacts their blood pressure resulting in hypertension (Wood, Goesling and Avellar, 2007).

Low fruits and vegetable consumption was found to be a significant predictor of hypertension among health workers in this study. Fruits and vegetable are a great source of potassium, which helps to prevent hypertension by maintaining renal homeostatic function (Wu and Wolley, 2019). A meta-analysis reported a similar association of low fruits and vegetable consumption with hypertension among the Asian population (Li et al., 2016). Previous studies in the Nepalese adult population found no association of fruits and vegetable consumption with hypertension (Dhungana et al., 2016; Khanal et al., 2017; Neupane et al., 2017). The unhealthy working environment (shift work, stress, high workload, unavailability of fruit and vegetable in the workplace) of the health 
workers force them to consume less fruit and vegetable than general people. The study setting of this study was in Kathmandu valley, Nepal, where unhealthy eating behaviours are in increasing trend, i.e., people prefer to eat easily available junk foods instead of fruits and vegetables (Resource Centre for Primary Health Care, 2016).

This study found the association between low physical activity and hypertension in health workers. Ibrahim stated that inadequate physical activity alters blood pressure homeostasis resulting in hypertension (Ibrahim, 2018). A consistent result was observed in the studies among the Nepalese population and Nigerian health workers (Chataut, Adhikari and Sinha, 2011; Dhungana et al., 2016; Sumaila et al., 2016). Biddle found that less physically active individuals experience more stress in their life, which is also a crucial inducer of hypertension (Biddle, 2016). Health workers of central hospitals in this study mainly use vehicles for transportation and deal with high workload with less or no physical movement makes them less physically active the whole day.

The current study found the association of alcohol use with hypertension among health workers. It was theoretically evident that alcohol use causes vasoconstriction action and alteration in the renin-angiotensin-aldosterone system, which ultimately leads to alcohol-induced hypertension (Crestani et al., 2014). With several reasons such as culturally acceptable in ethnic groups, peer pressure, stressful married life, family history of alcohol use, and smoking status, alcohol use is aggressively prevalent among the Nepalese population, especially in urban areas like Kathmandu. The percentage of alcohol use was found to be higher among the health workers $(59.20 \%)$ in this study than the national figure among Nepalese adult population $(26.5 \%)$ (Aryal et al., 2014). Based on the current findings, it was found that increased perceived stress among health workers direct them to consume more alcohol.

Regarding perceived stress, the current study found a significant association between increased perceived stress and hypertension. Research supported this association by stating that stress causes abnormal activation of the sympathetic nervous system alters hormonal cascades, which increases blood pressure, cholesterol levels, fibrinogen, and blood fluidity leads to cardiovascular events, including hypertension (Mucci et al., 2016). Previous studies among the general population demonstrated similar findings that the increased perceived stress was a significant factor of hypertension (Redmond et al., 2013; Bhelkar et al., 2018). The past studies in Indian health workers and Brazilian health workers observed consistent finding with the current research (Owolabi et al., 2012; Leonelli et al., 2017). Health workers have dual responsibility towards their work setting, and home laid them prone to get high perceived stress. The health workers in this study deal with the high flow of patients in hospitals and family issues, which lodged them to experience more stress.

This study is equipped with several strengths. Firstly, it is a novel study that uncovered the hidden burden of hypertension in Nepalese health workers. Secondly, health workers were benefited from this study as they had a chance to know their hypertensive status and take preventive measures on time. Lastly, study findings added up the health data of health workers that would help different scholars for further studies. Nevertheless, this study has a limitation on its study design. This study applied the cross-sectional study design, which cannot prove the real causal relationship of disease, but appropriate to determine the prevalence.

\section{CONCLUSION}

High prevalence of hypertension with its modifiable factors (educational level, fruits and vegetable consumption, 
physical activity, alcohol use, and perceived stress) and non-modifiable factors (age and marital status) among health workers were reported in the study. This study evident that health workers in Nepal are susceptible to hypertension as the general population, which might help to drag the attention of concerned bodies that health of health workers can deviate and priority of concern. It implies the urgent need for routine annual health examination, mainly focusing on the screening of blood pressure as well as effective interventions, including stress management strategies, lifestyle, and behavioural modification among Nepalese health workers. Knowledge, attitude, and practice regarding hypertension among Nepalese health workers can be a crucial part to investigate in further studies.

\section{REFERENCES}

Ahmed, M. T., Jadhav, J. and Sobagaiah, R. T. (2018) 'Assessment of risk factors of non- communicable diseases among healthcare workers in Nelamangala: A cross sectional study', International Journal of Community Medicine and Public Health, 5(2), pp. 745-748. doi: $10.18203 / 2394-$ 6040.ijcmph20180261.

Alghatrif, M. et al. (2013) 'Longitudinal trajectories of arterial stiffness and the role of blood pressure: The balitmore longitudinal study of aging', Hypertension, 62(5), pp. 934$941 . \quad$ doi: 10.1161/HYPERTENSIONAHA.113 .01445 .

Aryal, K. K. et al. (2014) Non communicable diseases risk factors: STEPS survey Nepal 2013. Kathmandu.

Asay, G. R. B. et al. (2016) 'Absenteeism and employer costs associated with chronic diseases and health risk factors in the US workforce', Preventing Chronic Disease, 13, pp.
1-11. doi: 10.5888/pcd13.150503.

Ban, A. (2018) 'Triple burden of women: Conflicting gender norms', The Himalayan Times, 2 March.

Bhelkar, S. et al. (2018) 'Association between stress and hypertension among adults more than 30 Years: A case-control study', National Journal of Community Medicine, 9(6), pp. 430-433.

Biddle, S. (2016) 'Physical activity and mental health: Evidence is growing', World Psychiatry, 15(2), pp. 176-177. doi: 10.1002/wps.20332.

Chataut, J., Adhikari, R. K. and Sinha, N. P. (2011) 'Prevalence and risk factors for hypertension in adults living in central development region of Nepal', Kathmandu University Medical Journal, 9(1), pp. 13-18. doi: 10.3126/kumj.v9i1.6255.

Chobanian, A. V et al. (2003) 'The seventh report of the joint national committee on prevention, detection, evaluation, and treatment of high blood pressure: The JNC 7 report', Jama, 289, pp. 2560-2572. doi: 10.1001/jama.289.19.2560.

Cohen, S. and Williamson, G. (1988) 'Perceived stress in a probability sample of the United States', in Spacapan, S. and Oskamp, S. (eds) The social psychology of health: claremont symposium on applied social psychology. Thousand Oaks, CA, US: Sage Publications, Inc, pp. 31-67.

Council For Technical Education and Vocational Training (2020) Programs of TSLC.

Crestani, C. C. et al. (2014) 'Cardiovascular alterations at different stages of hypertension development during ethanol consumption: time-course of vascular and autonomic changes', Toxicology and Applied Pharmacology. Elsevier Inc., 280(2), pp. 245-255. doi: 10.1016/j.taap.2014.08.012.

Dhungana, R. R. et al. (2016) 'Prevalence 
and associated factors of hypertension: A community-based cross-sectional study in municipalities of Kathmandu , Nepal', International Journal of Hypertension, 2016(236), pp. 1-10. doi: http://dx.doi.org/10.1155/2016/1656 938.

Ibrahim, M. M. (2018) 'Hypertension in developing countries: A major challenge for the future', Current Hypertension Reports. Current Hypertension Reports, 20(5), p. 38. doi: 10.1007/s11906-018-0839-1.

International Federation of Pharmaceutical Manufacturers and Associations (2016) Hypertension: putting the pressure on the silent killer.

Khanal, M. K. et al. (2017) 'Prevalence, associated factors, awareness, treatment, and control of hypertension: Findings from a cross sectional study conducted as a part of a community based intervention trial in Surkhet, Mid-western region of Nepal', PLoS ONE, 12(10), pp. 1-20. doi: 10.1371 /journal. pone.0185806.

Kibria, G. M. A. et al. (2018) 'Prevalence and associated factors of prehypertension and hypertension in Nepal: Analysis of the Nepal Demographic and Health Survey 2016', Health Science Reports, 1(10), p. e83. doi: 10.1002/hsr2.83.

Kretchy, I. A., Owusu-daaku, F. T. and Danquah, S. A. (2014) 'Mental health in hypertension: Assessing symptoms of anxiety, depression and stress on anti-hypertensive medication adherence', International Journal of Mental Health Systems, 8, p. 25. doi: 10.1186/1752-4458-8-25.

Leonelli, L. B. et al. (2017) 'Perceived stress among primary health care professionals in Brazil', Revista Brasileira de Epidemiologia, 20(2), pp. 286-298. doi: 10.1590/19805497201700020009.

Li, B. et al. (2016) 'Fruit and vegetables consumption and risk of hypertension: A meta-analysis', The Journal of Clinical Hypertension, 18(5), pp. 468-476. doi: 10.1111/jch.12777.

Manyisa, Z. M. and Aswegen, E. J. Van (2017) 'Factors affecting working conditions in public hospitals: A literature review', International Journal of Africa Nursing Sciences. The Authors, 6, pp. 28-38. doi: 10.1016/j.ijans.2017.02.002.

Ministry of Health and Population (2013) Human resource for health Nepal country profile.

Mucci, N. et al. (2016) 'Anxiety, stressrelated factors, and blood pressure in young adults', Frontiers in Psychology, 7, p. 1682. doi: 10.3389/fpsyg.2016.01682.

Muzzi, M., Pawlina, C. and Schnorr, G. P. (2018) 'Prevalence of stress in health workers in the context hospital', Psychology and Behavioral Medicine Open Access Journal, 0(0), pp. 15-21.

Naing, L., Winn, T. and Rusli, B. N. (2006) 'Pratical issues in calculating the sample size for prevalence studies', Archives of Orofacial Sciences, 1, pp. 9-14.

Neupane, D. et al. (2014) 'Prevalence of hypertension in member countries of South Asian Association for Regional Cooperation (SAARC): Systematic review and meta-analysis', Medicine, 93(13), p. e74. doi: 10.1097/MD.0000000000000074.

Neupane, D. et al. (2017) 'Awareness, prevalence, treatment, and control of hypertension in western Nepal', American Journal of Hypertension, 30(9), pp. 907-913. doi: 10.1093/ajh/hpx074.

NH Department of Administrative Services (2014) Perceived stress scale.

Osei-yeboah, J. et al. (2018) 'Cardiometabolic risk factors among healthcare workers: A cross-sectional study at the Sefwi-Wiawso municipal hospital, Ghana', Biomedical Research International, 2018(3), pp. 
1-9. doi: $10.1155 / 2018 / 8904548$.

Owolabi, A. O. et al. (2012) 'Work-related stress perception and hypertension amongst health workers of a mission hospital in Oyo', African Journal of Primary Health Care and Family Medicine, 4(1), pp. 1-7. doi: 10.4102/phcfm.v4i1.307.

Redmond, N. et al. (2013) 'Perceived stress is associated with incident coronary heart disease and all-cause mortality in low-but not high-income participants in the reasons for geographic and racial differences in stroke study', Journal of the American Heart Association, 2(6), p. e000447.

doi:

10.1161/JAHA.113.000447.

Resource Centre for Primary Health Care (2016) A research on: The food environment of Kathmandu metropolitan area 2016. Kathmandu.

Sahebi, L., Vahidi, R. G. and Mousavi, S. H. (2010) 'Prevalence of Hypertension and Associated Variables in Hospital Staff in Iran', Acta Medica Saliniana, 39(1), pp. 6-13. doi: 10.5457/ams.138.10.

Schwenk, T. L. (2017) Lower education level is associated with higher risk for cardiovascular disease, Jama Internal Medicine.

Sirinara, P., Hanprathet, N. and Jiamjarasrangsi, W. (2019) 'Prevalence of hypertension and associated factors among healthcare workers: A cross-sectional study', Chulalongkorn Medical Journal, 63(3), pp. 193-199. doi: 10.14456/clmj. 10.

Sumaila, F. G. et al. (2016) 'Prevalence of undiagnosed hypertension and its risk factors among health care workers of some selected hospitals in Dutse, Jigawa State, North western Nigeria', Advances of Science for Medicine, 1(2), pp. 19-23. doi: 10.5281/zenodo.1308458.

Taylor, M. and Bithoney, W. (2012) 10 Steps to developing a culture of health for hospital and health system employers [White paper].

Thapa, D., Malla, G. and KC, A. (2018) 'Sleep quality and related health problems among shift working nurses at a tertiary care hospital in eastern Nepal: A cross sectional study', Journal of Nursing and Health Studies, 2(3), pp. 1-4. doi: 10.21767/2574-2825.100029.

Wood, R. G., Goesling, B. and Avellar, S. (2007) The effects of marriage on health: A synthesis of recent research evidence. Princeton, NJ.

World Health Organization (2013) World health day: High blood pressure global and regional overview.

World Health Organization (2017) Protecting workers health [Fact Sheet].

World Health Organization (2018) Non communicable disease country profiles 2018.

$\mathrm{Wu}, \mathrm{A}$. and Wolley, M. (2019) 'The interplay of renal potassium and sodium handling in blood pressure regulation: Critical role of the WNKSPAK-NCC pathway', Journal of Human Hypertension, 33(7), pp. 508-523. doi: 10.1038/s41371-0190170-6. 